\title{
The Relationship of Emotional Stability with Job Conscience and Professional Commitment
}

\author{
Seyed Fakhr Al-din Hossieni ${ }^{1}$, Faezeh Soltani ${ }^{2, *}$
}

${ }^{1}$ MSc, Rafsanjan University of Medical Sciences, Rafsanjan, Iran

${ }^{2}$ MSc, Kerman University of Medical Sciences, Kerman, Iran

* Corresponding author: Faezeh Soltani, MSc, Kerman University of Medical Sciences, Kerman, Iran. E-mail: sfaezeh14@yahoo.com

Received: 17 Oct 2017

Accepted: $27 \mathrm{Feb} 2018$

\begin{abstract}
Introduction: Emotional stability is a personality trait that includes natural and constant domains of emotions found in individuals at different stages of life and in different environments. This study aimed at determining the relationship of emotional stability with job conscience and professional commitment.
\end{abstract}

Methods: This study had a descriptive-analytical design. This study was conducted on 200 nurses of Kerman University of Medical Sciences through cluster random sampling. Data was gathered using a questionnaire, which included 4 parts: demographic data, Goldberg emotional stability questionnaire, Mac Cry Job conscience questionnaire, and Henning and Clikeman professional commitment questionnaire. Data were analyzed with the SPSS version 20 software using Pearson correlation test, independent T-test, Analysis of Variance (ANOVA), and multiple linear regression. Results: The mean score of emotional stability, Job conscience, and professional commitment among nurses was $46.2( \pm 15.6), 44.4( \pm 14.3)$, and $49( \pm 14.4)$. Forty percent of nurses had high emotional stability. There was a statistically significant positive correlation between emotional stability with Job conscience $(\mathrm{P}<0.0001)$ and professional commitment $(\mathrm{P}<0.00001)$. There was also a statistically significant positive correlation between Job conscience and professional commitment $(\mathrm{P}<0.0001)$.

Conclusions: According to the results of the present study, there was a positive correlation between emotional stability and job conscience, and professional commitment. Therefore, attention to emotional stability, training and planning for its growth, creating corporate profits, and improving professional commitment and job conscience is required.

Keywords: Emotional Stability, Job Conscience, Professional Commitment, Nurse

(C) 2018 Iranian Nursing Scientific Association (INA) 


\title{
بررسى ارتباط ثبات عاطفى با وجدان كارى و تعهد حرفهاى يرستاران شافل در

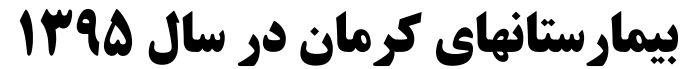

\author{
سيد فخرالدين حسينى ' فائزه سلطانى r.**
}

$$
\begin{aligned}
& \text { ' كارشناس ارشد، كروه يرستارى، دانشعاه علوم يزشكى رفسنجان، رفسنجان، ايران }
\end{aligned}
$$

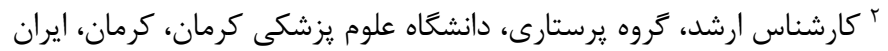

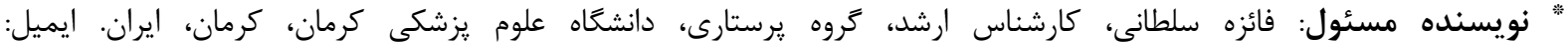

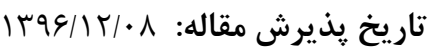
sfaezeh14@yahoo.com

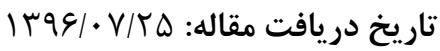

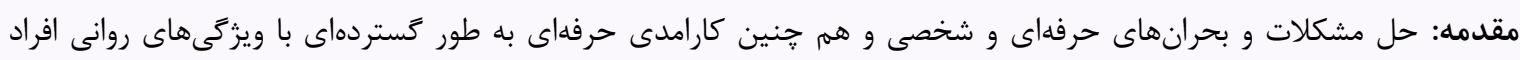

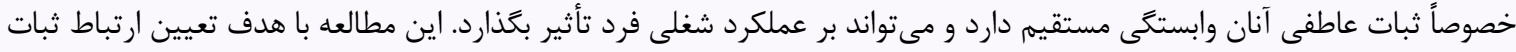

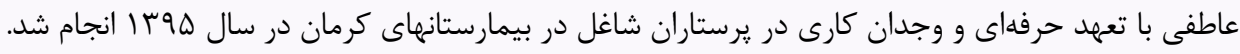

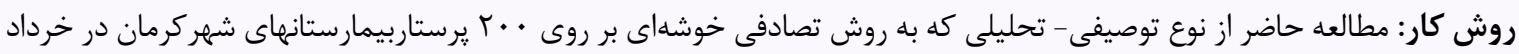

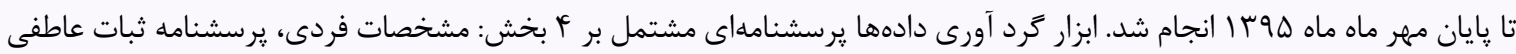

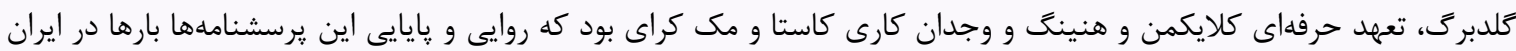

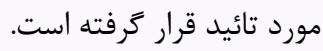

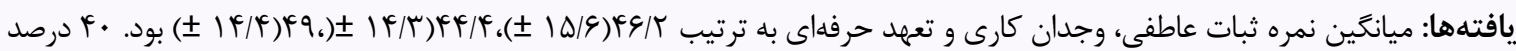

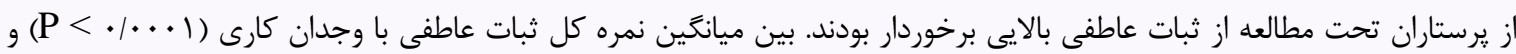

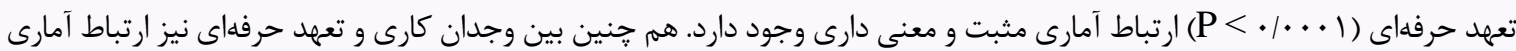

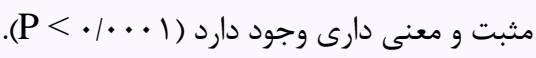

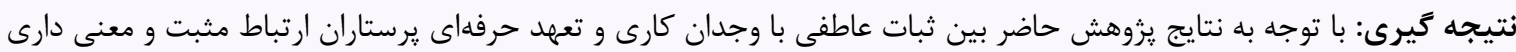

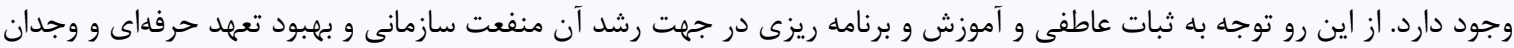
كارى را به همراه دارد. وازَّان كليدى: ثبات عاطفى، وجدان كارى، تعهد حرفهاى، يرستار

بلندمدت و ظرفيت تحمل تأخير وتجديد نظر در انتظارات خود را دارند. فرد

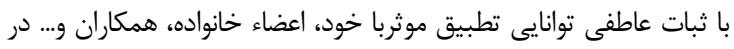

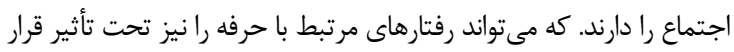

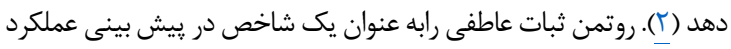

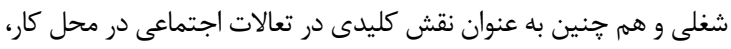

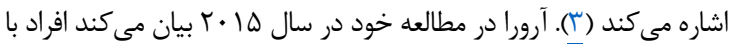

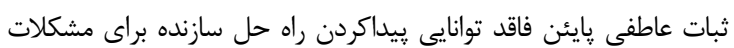

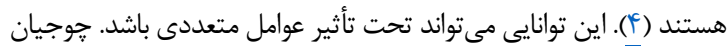

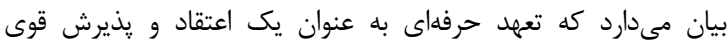

\section{مقلهمه}

انجام مراقبت و اجراى مداخلات يرستارى مهمهرين و اصلىترين وظيفه

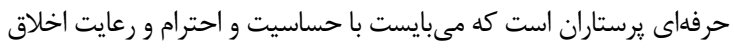

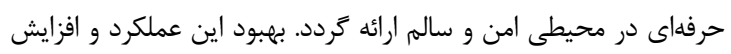

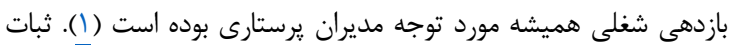

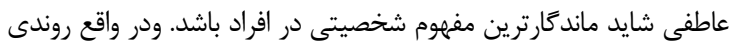

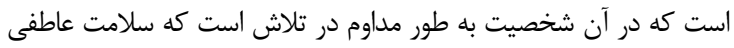

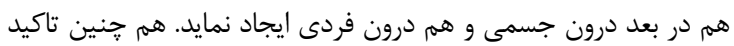

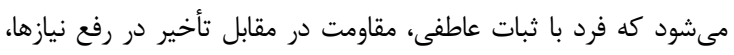

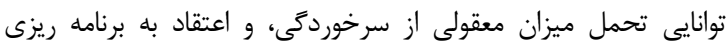




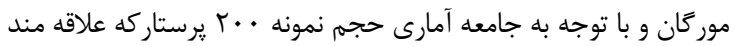

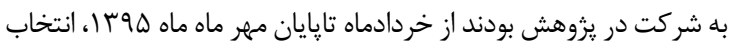

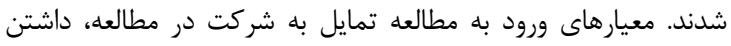

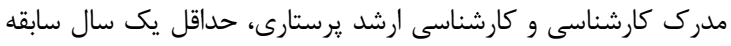

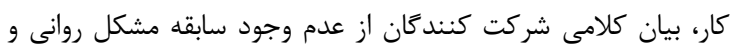

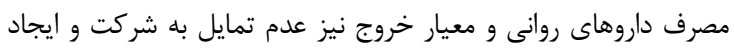

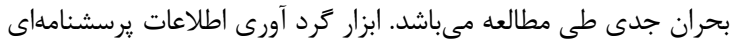

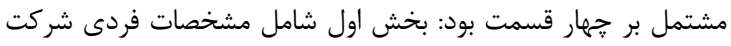
كنندكان شامل (سن، جنس، وضعيت تأهل، سطح تحصيلات، نوبت فيت

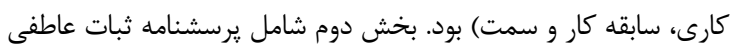

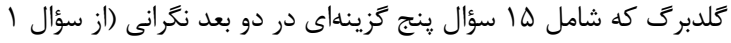

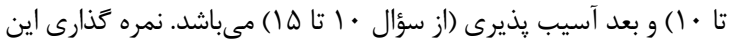

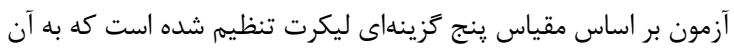

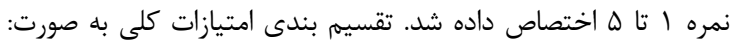

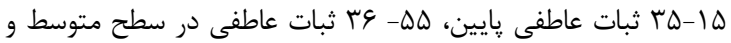
هن

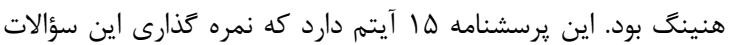

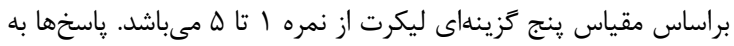

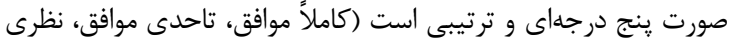

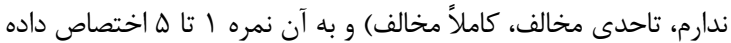

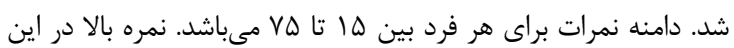

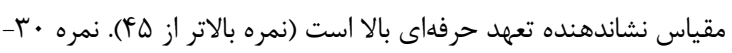

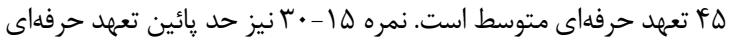

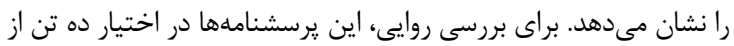

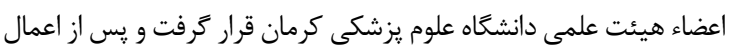

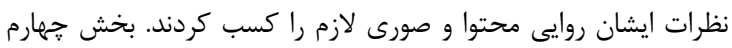

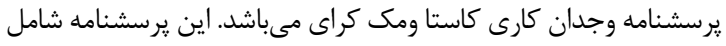

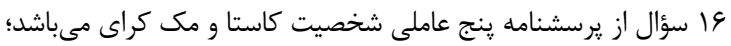

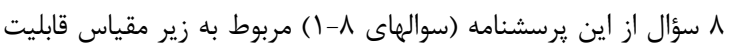

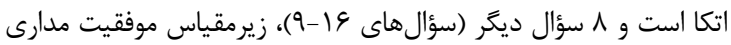

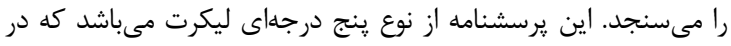

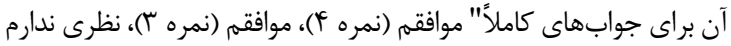

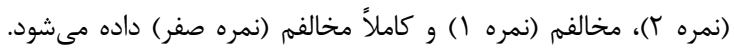

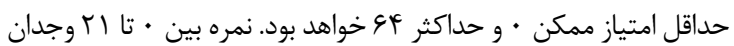

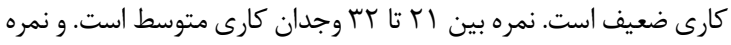

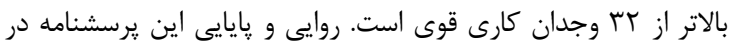

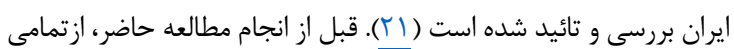
يرستاران رضايت شفاهى اخذ شد و به ايشان اطمينان داده شد كه دادهها

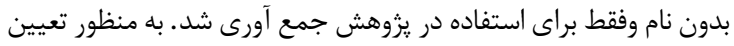

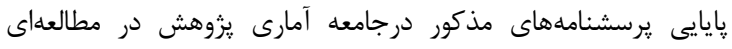

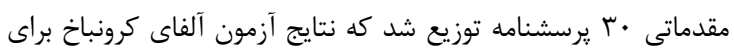

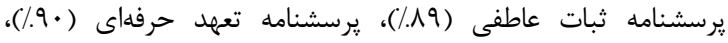

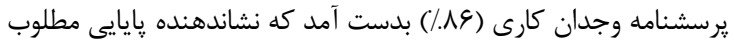

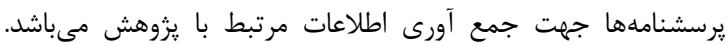

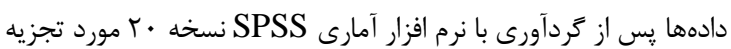

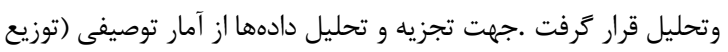

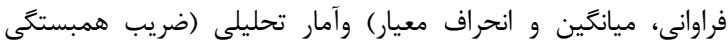

ارزشهاى حرفهاى، تمايل به تلاش بسيار جهت ارتقاى حرفه و حرفهاى

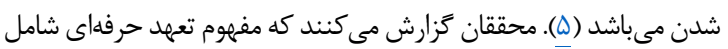

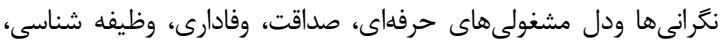

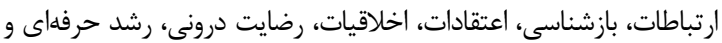

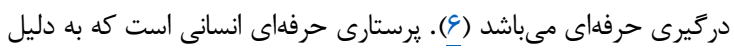

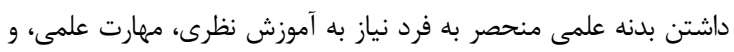

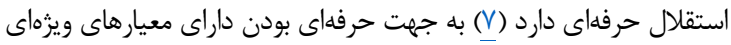

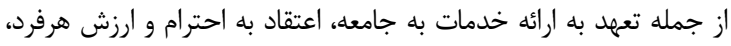

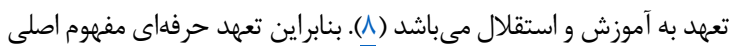

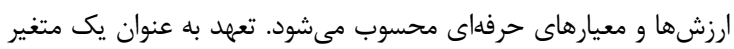

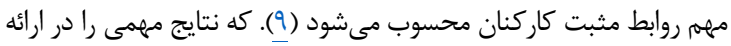

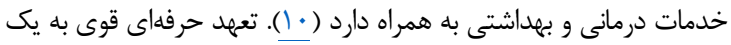

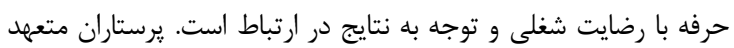

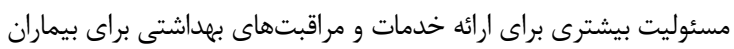

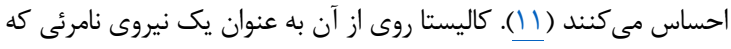

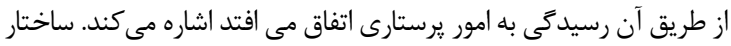

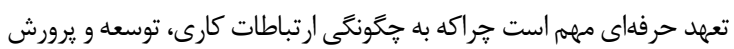

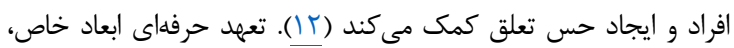
تعار يف متفاوت و حالشهاى متعدد دارد. در مطالعه جعفر آقايى نشان داد

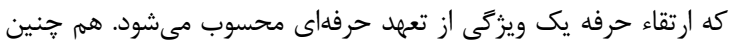

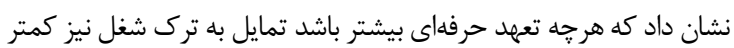

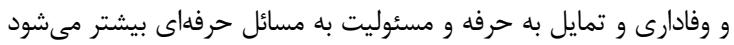

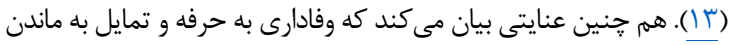

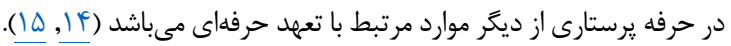

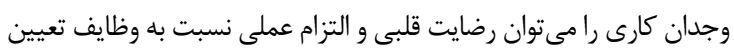

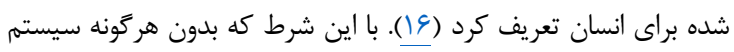

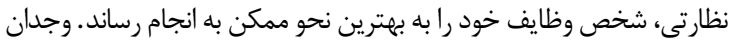

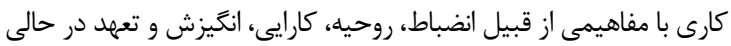

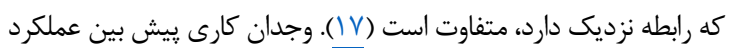

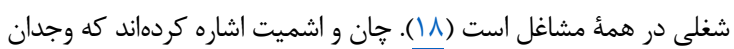

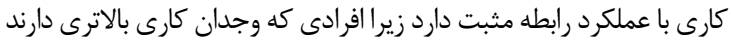
وقت و تلاش بيشترى را براى انجام تكليف خود صرف مي كنيند (9 (1). ميلر

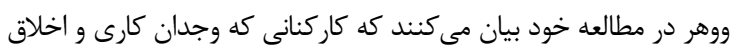

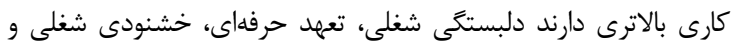

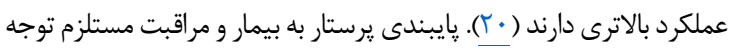

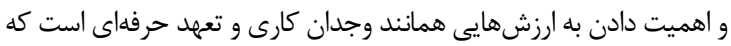

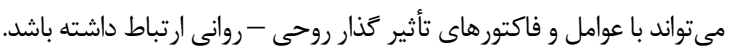
وجدان كارى ييش شرط مهم تحقق هر نوع برنامه ريزى در محيط كار

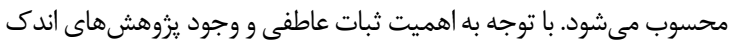

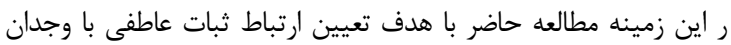

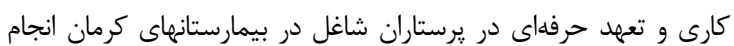

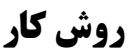

يروهش حاضر يك مطالعه مقطعى از نوع همبستَى مى باشد. نمونه ََيرى

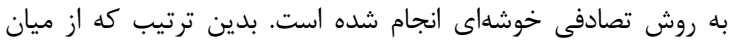

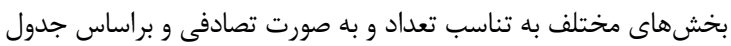


بودند. ساير مشخصات دموكرافيك افراد تحت مطالعه در جدول إ آمده است. ميانكين و انحراف معيار ثبات عاطفى آ

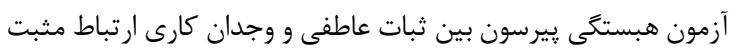

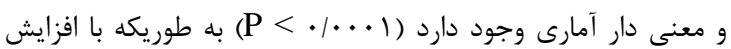

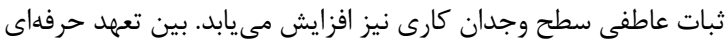

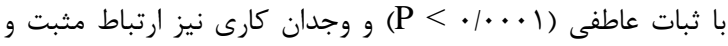

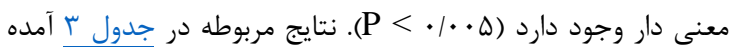

קيرسون، t مستقل وركرسيون گام به كام Anova .... استفاده شد. به

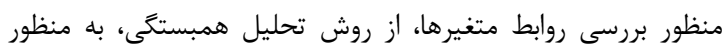

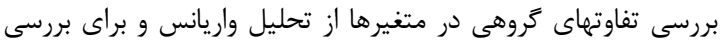

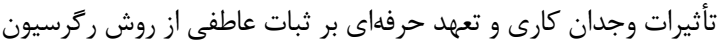

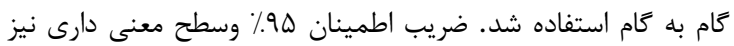
(P< > نظر كرفته شد.

\section{كافته ها}

ميانكين و انحراف معيار سنى يرستاران شركت كننده در اين يزوهش

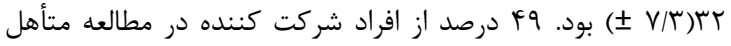

جدول ا: اطلاعات فردى يرستاران تحت مطالعه

\begin{tabular}{|c|c|}
\hline تعداد (درصد) & متغير \\
\hline & جنس \\
\hline $\mathbb{I r} \cdot(\xi \cdot)$ & 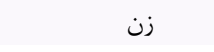 \\
\hline \multirow[t]{2}{*}{$(\boldsymbol{q} \cdot) \Lambda}$. & مرد مرد \\
\hline & وضعيت تأهل \\
\hline$(89) 91$ & متأهل \\
\hline$(\mathcal{E T}) \wedge \varepsilon$ & مجرد \\
\hline \multirow[t]{2}{*}{$(\wedge) 19$} & ساير \\
\hline & سابقه كار \\
\hline$(\Delta)) \cdot r$ & • •-1 سال \\
\hline$(r \varepsilon / \Delta) V r$ & •l-1·r سال \\
\hline \multirow[t]{2}{*}{$(I r / \Delta) \Gamma \Delta$} & 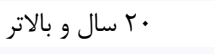 \\
\hline & نوبت كارى \\
\hline$(\varepsilon \wedge / Q)) T V$ & در كردش \\
\hline \multirow[t]{2}{*}{ س } & ثابت \\
\hline & سمت \\
\hline (^I)IGY & يرستار \\
\hline$(1 / / \Delta) r r$ & سريرستار \\
\hline \multirow[t]{2}{*}{$(V / \Delta) \backslash \Delta$} & سويروايزر \\
\hline & سطح تحصيلات \\
\hline$(A T) \backslash G Y$ & ل ليسانس \\
\hline$(1 \wedge) r^{4}$ & فوق ليسانس \\
\hline
\end{tabular}

جدول r: فراوانى و درصد ثبات عاطفى، وجدان كارى و تعهد حرفهاى برستاران شركت كننده در مطالعه

\begin{tabular}{|c|c|c|}
\hline \multirow[t]{2}{*}{ درصد } & فراوانى & دامنه نمرات \\
\hline & & وجدان كارى \\
\hline $1 T / Q$ & ra & كم \\
\hline $1 \Delta / \Delta$ & r & متوسط \\
\hline \multirow[t]{2}{*}{ VT } & IfF & بالا \\
\hline & & ثبات عاطفى \\
\hline$F 1 / 0$ & ᄉr & ضعيف \\
\hline $1 N / \Delta$ & $r v$ & متوسط \\
\hline \multirow[t]{2}{*}{$r$} & $\wedge$. & قوى \\
\hline & & تعهد حرفهاى \\
\hline ry & 91 & يائين \\
\hline $1 / \Delta$ & $r$ & متوسط \\
\hline$s 4 / 0$ & $1 r 9$ & بالا \\
\hline
\end{tabular}


بخشهاى مختلف از آزمونهاى شخصيتى استفاده شود تا به منفعت

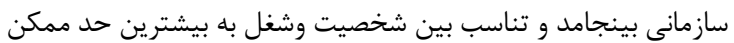

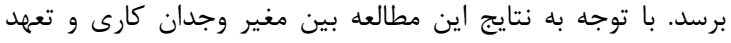

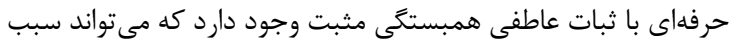

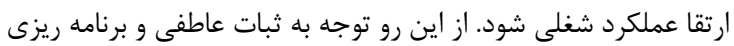

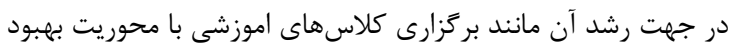

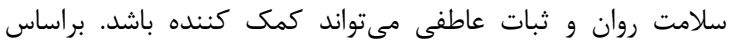

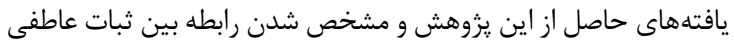
با وجدان كارى و تعهد حرفهاى، مديران يرستارى و سياست كزاران

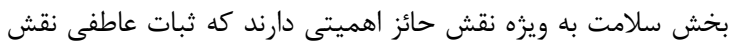

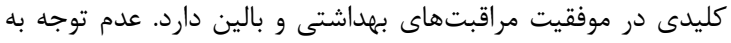

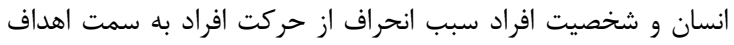

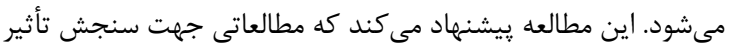
آموزش و برنامههاى ضن خدمت با محوريت ثبات عاطفى انجام شود. سياسگزارى يزوهشكران بر خود لازم مىدانند از كليه عزيزانى كه ما را در انجام

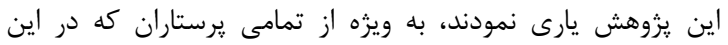

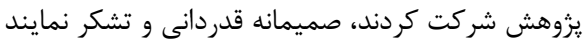

\section{References}

1. Ahadi B, Fathi A, Abdalmohammadi K. Study of psychological Factors Affecting Job Performance East Azerbaijan Police Command Staff. J Police Knowledge Azerbaijan East. 2014;4(15):63-78.

2. Brose A, Scheibe S, Schmiedek F. Life contexts make a difference: emotional stability in younger and older adults. Psychol Aging. 2013;28(1):148-59. DOI: 10. 1037/a0030047 PMID: 23066802

3. Judge TA, Van Vianen AE, De Pater IE. Emotional stability, core self-evaluations, and job outcomes: A review of the evidence and an agenda for future research. Hum Perform. 2004;17(3):325-46.

4. Arora R, Rangnekar S. Relationships Between Emotional Stability, Psychosocial Mentoring Support and Career Resilience. Eur J Psychol. 2015;11(1):1633. DOI: 10.5964/ejop.v11i1.835 PMID: 27247639

5. Chouh J, Hsiao-Chiao W, Tsai-Chung L, Li-Chi H. Reliability and validity of nurses' job satisfaction scale and nurses professional commitment. Taichung, Taiwan2007.

6. Goodarzvand M, Amin Z. The Relationship between Gender and Organizational Commitment in public Organizations Considering Age. Q Soc Woman. 2013;3(4):43-68.

7. Karimi D. The Study of Relation Between Personal Characteristics and Police Occupational Performance. Police Organ Dev J. 2009;6(30):25-39.

8. Lemmens K, Strating M, Huijsman R, Nieboer A. Professional commitment to changing chronic illness care: results from disease management programmes. Int J Qual Health Care. 2009;21(4):233-42. DOI: 10. 1093/intqhc/mzp017 PMID: 19389724

9. Forouzande R, Ganji F, Nekoei A, Nematei S. Leadership effectiveness and its relationship with emotional stability among nurse managers in

$$
\begin{aligned}
& \text { يزوهش افرادى تحت مطالعه قرار كَرفتند كه با انسان و نه ابزار و اشياء }
\end{aligned}
$$

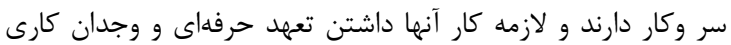

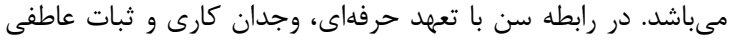

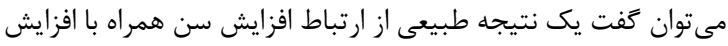

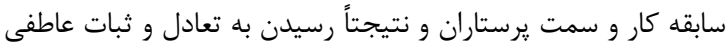

$$
\begin{aligned}
& \text { مىباشد. ارتباط تعهد حرفهاى و وجدان كارى برى باسن با مطالعه آنجليا }
\end{aligned}
$$

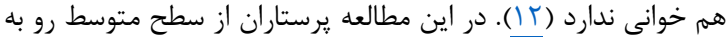

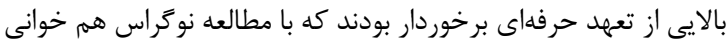

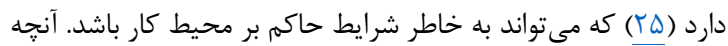

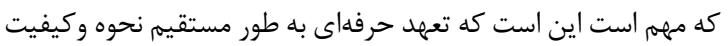

$$
\begin{aligned}
& \text { انجام كار در سازمانها و به ويزّه حرفه يرستارى كه با با روح و و جانانسان }
\end{aligned}
$$

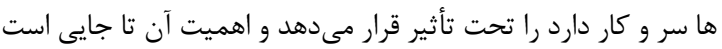

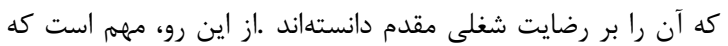

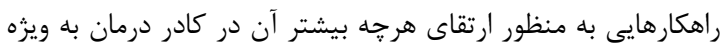

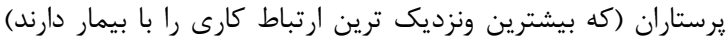

$$
\begin{aligned}
& \text { وِيش بينى و اجرا كرد. } \\
& \text { نتيجه تيرى }
\end{aligned}
$$

با جمع بندى نتايج بالا به مديران يرستارى در سطح كلان مىتوان

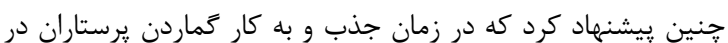

educational hospitals related to Isfahan University of Medical Science in 2007. J Clin Nurs Midwifery. 2013;2(3):64-73.

10. Nabizadeh Gharghozar Z, Atashzadeh Shoorideh F, Khazaei N, Alavi-Majd H. Assessing organizational commitment in clinical nurses. Q J Nurs Manage. 2013;2(2):41-8.

11. Niazazari K, Enayati T, Behnamfar R, Kahroodi Z. Relationship between Professional Ethics and Job Commitment. Iran J Nurs. 2014;27(87):34-42.

12. Sutin AR, Terracciano A, Ferrucci L, Costa PT, Jr. Teeth Grinding: Is Emotional Stability related to Bruxism? J Res Pers. 2010;44(3):402-5. DOI: 10.1016/j.jrp.2010.03.006 PMID: 20835403

13. Terracciano A, Lockenhoff $\mathrm{CE}$, Zonderman $\mathrm{AB}$, Ferrucci L, Costa PT, Jr. Personality predictors of longevity: activity, emotional stability, and conscientiousness. Psychosom Med. 2008;70(6):6217. DOI: 10.1097/PSY.0b013e31817b9371 PMID: $185 \overline{96250}$

14. Alirezaei N, Massah N, Akrami H. The Relation between Work Conscientiousness with Job Performance. Ethics Sci Technol. 2013;8(2):1-12.

15. Ahmadi P, Babashahi J, Khoda Shenas L. Considering the Relationship Between Personality Factors and Entrepreneurship: A Research About The Personnel of Sadad Informatics Corporations. Res Manage Organ Resources. 2013;1(3):1-21.

16. Bakhshi Sourshajani L. The Relationship between Emotional Intelligence, Mental Health, and the Organizational Commitment among High School Teachers, Hospital Nurses, and the Water and Power Company Personnel in Behbahan. J Mod Thoughts Educ. 2011;6(1):65-81. 
17. Costa P, McCrae R. The Revised NEOPersonality Inventory (NEOPI-R). In: Saklofske D, editor. Handbook ofpersonality theory and testing. London: Sage Publications; 1992.

18. Gellatly W, Irving A. The role ofconscientiousness in industrial context. Long Range Plan. 2001;33:35-54.

19. Farrokhi M. The relationship between organizational culture and organizational commitment, and job conscience Azad University of Babol. J Educ Manage. 2016;7(2):96-118.

20. Mckinney Arlise P. Goel orientation: a test of competing models. Virginia Virginia Polytechnic Institute and State University; 2003.

21. Hosseini M, Jahangir Fard M. The relationship between leadership style and work ethic and organizational commitment. 2012;3(18):34-49.
22. Barati Ahmadabadi H. Oreizi Relationship between organization climate and work conscientiousness with job performance. J Appl Psychol. 2013;1(13):65-81.

23. Brown C, George-Curran R, Smith ML. The Role of Emotional Intelligence in the Career Commitment and Decision-Making Process. J Career Assess. 2016;11(4):379-92. DOI: 10.1177/1069072703255 $\underline{834}$

24. Moradi M, Khatooni M, Jahani Hashemi H, Zeighami R, Sheikh M. Relationship between Professional Commitment and Job Satisfaction in Qazvin's Pubic Educational Hospital Nurses. J Med Ethics 2013:5678.

25. Nogueras DJ. Occupational commitment, education, and experience as a predictor of intent to leave the nursing profession. Nurs Econ. 2006;24(2):86-93, 55. PMID: 16676751 